\title{
Le "Discours de la servitude volontaire" et la cause protestante: les paradoxes de la réception de La Boétie
}

\section{Philippe Desan}

\section{(2) OpenEdition}

Journals

\section{Édition électronique}

URL : http://journals.openedition.org/studifrancesi/9759

DOI : 10.4000/studifrancesi.9759

ISSN : 2421-5856

Éditeur

Rosenberg \& Sellier

\section{Édition imprimée}

Date de publication : 1 août 2017

Pagination : 211-222

ISSN : 0039-2944

\section{Référence électronique}

Philippe Desan, «Le "Discours de la servitude volontaire" et la cause protestante: les paradoxes de la réception de La Boétie », Studi Francesi [En ligne], 182 (LXI | II) | 2017, mis en ligne le 01 août 2018, consulté le 05 janvier 2021. URL : http://journals.openedition.org/studifrancesi/9759; DOI : https:// doi.org/10.4000/studifrancesi.9759

\section{(c)}

Studi Francesi è distribuita con Licenza Creative Commons Attribuzione - Non commerciale - Non opere derivate 4.0 Internazionale. 


\section{Le "Discours de la servitude volontaire" et la cause protestante: les paradoxes de la réception de La Boétie}

\section{Abstract}

There is a paradox in La Boétie's Discourse on volontary servitude between the primary intention of the author and the reception of the text by its readers during the religious wars. It is therefore necessary to distinguish between the time of its drafting and the time of its publication. The interpretative difficulty inherent to the Discourse facilitated its cooptation by the protestant reformers. Read in the religious context of the post-Saint-Bartholmew's Day massacre, the Discourse acquired a seditious dimension it did not originally have. Like Luther before him, La Boétie defends a conception of liberty that is more spiritual than material, but the Monarchomachs transformed this «youthful exercise» into a call for armed uprising, not by the people directly but via the increasing power of the magistrates.

Le Discours de la servitude volontaire d'Estienne de La Boétie demeure encore aujourd'hui un texte majeur dans l'histoire de la philosophie politique. Certes, cet "essai" reste d'actualité et est pour cette raison fréquemment lu hors du contexte immédiat de sa rédaction et de sa publication. Cette approche atemporelle et essentiellement philosophique ne permet pourtant pas de saisir les enjeux de sa réception et de ses récupérations politiques au temps des guerres de religion. Le Discours présente de plus une particularité inhabituelle pour un texte politique de la Renaissance, à savoir le décalage entre ce que nous pourrions appeler l'intention première de son auteur et la façon dont il fut reçu par ses premiers lecteurs. Il nous parait donc important d'établir une distinction entre le temps de la rédaction du Discours de la servitude volontaire et le temps de sa publication, et conséquemment de sa première réception. Ces deux moments sont séparés par au moins une quinzaine d'années - voire vingt -, c'est-à-dire une génération. Le début des guerres de religion (que l'on peut dater du massacre de Vassy en mars 1562) représente un événement majeur dans la réception du Discours. En fait, on pourrait arguer que le conflit religieux qui divise la France à partir des années 1560, et plus particulièrement le Sud-Ouest de la France, entraîna une réception du Discours à la fois inattendue et paradoxale. La difficulté d'interpréter ce texte facilita son détournement au service de la cause protestante.

On s'est justement demandé si le Discours n'avait pas été conçu comme une réponse à un événement historique précis. Un contemporain de Montaigne et de La Boétie, Jacques-Auguste de Thou, soutient par exemple que l'auteur du Discours aurait voulu réagir à la répression brutale qui avait fait suite à la révolte de la gabelle en 1548 à Bordeaux, critiquant ainsi ouvertement la répression sanglante du connétable de Montmorency ${ }^{1}$. Malgré l'importance politique de ce soulèvement du peuple

(1) Sur la révolte de la Gabelle à Bordeaux, voir S.C. GIGON, Contribution à l'bistoire de l'impôt sous l'ancien régime. La révolte de la Gabelle en Guyenne (1548-1549), Paris, H. Champion, 1906. On consultera également les relations et les commentaires relatifs à cet événement par des contemporains de La Boétie: G. Paradin, La Révolte de la gabelle en Guienne l'an mil cinq cens quarante-buit, tirée de l'Histoire 
à Bordeaux et le traumatisme de ses conséquences brutales parmi la population et les lettrés de ce temps, il est pourtant difficile de voir dans cet événement la seule origine du traité de La Boétie. Quand on critique un événement de cet ordre, on s'y prend différemment - surtout à une époque où les pamphlets séditieux et ouvertement engagés ne manquent pas -, à moins de croire que La Boétie redoutait d'être persécuté et aurait ainsi choisi d'offrir une critique voilée et cryptée des pratiques du pouvoir. C'est par exemple la thèse soutenue par les straussiens².

Dans Persecution and the Art of Writing (1952), Leo Strauss (1899-1973) part du principe que, dans la crainte d'être persécutés pour leurs idées, la plupart des grands auteurs avant le XVIII siècle auraient développé des façons détournées pour exprimer leurs idées. Ce langage caché - réclamant une lecture entre les lignes - aurait profondément influencé et même défini leur style tout en permettant aux «lecteurs suffisants» d'y déceler des critiques voilées des pratiques politiques de leur époque. Les suiveurs de Léo Strauss ont vu en La Boétie un des meilleurs exemples de cet art de la dissimulation pour le XVI ${ }^{\mathrm{e}}$ siècle; certains allant même jusqu'à avancer que le Discours aurait été rédigé par Montaigne lui-même ${ }^{3}$. Parmi les critiques modernes, on s'est donc intéressé à cet événement marquant - peut-être la célèbre «Malencontre» dont parle La Boétie - qui aurait entraîné la rédaction du Discours. À partir de cette conception événementielle de l'histoire, tout un courant interprétatif s'est concentré sur la recherche de l'“étincelle" qui aurait en quelque sorte déclenché la rédaction du Discours.

Il n'est certes pas faux de dire que le Discours possède sa propre historicité, au même titre que tout autre texte d'ailleurs. On sait par exemple qu'il fut remanié à plusieurs reprises par son auteur: les éloges de Ronsard, Baif et Du Bellay sont des ajouts probablement postérieurs à 1552 . Guy Demerson a émis l'hypothèse que les adresses à Longa ont été ajoutées en 1554 lors de la bataille de l'édit du Semestre, c'est-à-dire au moment de la nomination de La Boétie au parlement de Bordeaux et de Lur Longa au parlement de Paris ${ }^{4}$. On sait également que le texte circula sous forme manuscrite du vivant de La Boétie ainsi qu'après sa mort en $1563^{5}$. Pourtant,

de notre tems de maitre Guillaume Paradin, Lyon, Pierre Michel, 1558; et J. BouchET, Histoire de la Révolte de la Gabelle en Guyenne et à Bordeaux [...] tirée des Annales d'Aquitaine, Poitiers, Enguilbert de Marnef, 1557. Ces textes sont reproduits dans La Révolte de la gabelle en Guyenne et à Bordeaux en 1548, Bordeaux, Atelier Aldo Manuzio, 1981.

(2) Voir Ph. Desan, Une lecture straussienne des "Essais", ou le Montaigne du docteur Armaingaud, «Bulletin de la Société Internationale des Amis de Montaigne» 56, 2012, pp. 55-68.

(3) D. Schaeffer, The Political Philosophy of Montaigne, Ithaca, Cornell University Press, 1990; Freedom over Servitude: Montaigne, La Boétie, and On Voluntary Servitude, Westport, Greenwood Press, 1998.

(4) G. DEMERSON, «Les exempla dans le Discours de la servitude volontaire: une rhétorique datée?», dans M. Tetel (dir.), Étienne de la Boétie. Sage révolutionnaire et poète périgourdin, Paris, H. Champion, 2004, pp. $195-224$.

(5) Dès les années 1550, le Discours semble avoir circulé dans plusieurs pays européens. Une copie a récemment été découverte à la bibliothèque Folger-Shakespeare de Washington (de provenance anglaise) et une autre à la bibliothèque Ambrosiana de Milan. Voir M. SCHACHTER, Presentation of a newly Discovered Manuscript of La Boétie's "Discours de la servitude volontaire" and Hypotheses on the Datation of the BnF Manuscripts, «Montaigne Studies», vol. XX, 2008, pp. 185-206; et J.-E. GIROT, Une version inconnue du "Discours de la servitude volontaire" de La Boétie, «Bibliothèque d'Humanisme et Renaissance» 3, t. 63, 2001, pp. 551-565. D'autres copies manuscrites du Discours sont conservées dans des bibliothèques françaises, dont une à la BnF dans la collection Dupuy. Parmi les nombreuses études qui retracent l'histoire du Discours de la servitude volontaire, voir notamment R. RAGGHIANTI, "Discours de la servitude volontaire" et Étienne de La Boétie: d'une énigme à l'autre, «Rinascimento», vol. XLIII, 2003, pp. 507-552; ID., Rétablir un texte. Le "Discours de la servitude volontaire" d'Étienne de La Boétie, Florence, Leo Olschki, 2010. Pour une bibliographie raisonnée complète (jusqu'en 1997) sur La Boétie et le Discours, voir M. MAGNIEN, Étienne de La Boétie, Paris/Rome, Memini, coll. «Bibliographie des Écrivains Français», 1997. Plus récemment, Déborah KNop et Jean BALSAMo ont fait le point sur la diffusion du Discours avant sa première impression au début des années 1570: voir "De la servitude volontaire". Rhétorique et politique en France sous les derniers Valois, Rouen, Presses Universitaires de Rouen et du Havre, 2014. 
force est de constater que nous ne connaissons pas avec certitude les intentions de La Boétie; le texte lui-même n'offrant aucune référence précise à ce sujet. Que son texte s'inscrive dans l'histoire de son temps est une chose, mais dire que son objet est de répondre directement à un événement précis représente une extrapolation hâtive dans la mesure où des doutes subsistent sur la date de sa rédaction.

Si le moment de la rédaction demeure incertain, celui de sa transmission est par contre plus facile à analyser. C'est pourquoi nous proposons de dissocier le temps de l'écriture du temps de la réception imprimée du Discours et d'étudier uniquement la réception protestante du texte de La Boétie sans chercher à relier les intentions inconnues de l'auteur à la compréhension et à l'interprétation, vingt ans plus tard, de ce texte alors que les guerres de religion font rage en France. Les livres ont des vies qui échappent parfois au contrôle de leurs auteurs et le Discours tombe précisément dans ce cas de figure. L'erreur serait de "forcer" absolument un rapprochement entre ces deux moments - l'écriture et la réception imprimée - pour tenter de découvrir une logique qui expliquerait le paradoxe d'une lecture protestante du Discours. Nous n'aborderons pas non plus le problème de la circulation du Discours sous forme manuscrite au sein d'un petit réseau d'initiés et de lettrés dans les années 1550 , car c'est là un tout autre problème. Ce qui retient notre attention ici c'est l'existence imprimée du Discours dans le contexte des guerres civiles. Nous n'émettrons aucune suggestion sur ce que ce texte tente d'accomplir, et n'aborderons que rapidement son message politique et idéologique évident pour certains et cryptique pour d'autres. Notre approche se veut sociologique - dans la tradition d'une sociologie de la littérature - et traite exclusivement du Discours dans sa matérialité: un livre lu et interprété d'une certaine façon par les réformés qui virent en La Boétie un des hérauts de la cause protestante.

Projetons-nous au début des années 1570. En 1572, le massacre de la SaintBarthélemy modifia grandement la réception du texte de La Boétie en faisant passer le Discours pour un pamphlet réformé. Pour comprendre l'évolution idéologique du Discours, il est essentiel de contextualiser dans la micro-durée le temps de la transmission imprimée du Discours. En fait, l'histoire de la réception du Discours dépasse largement l'intention première de l'auteur, quelle qu'elle fût, car il ne faut pas perdre de vue que l'existence livresque du Discours est strictement protestante.

Comme on le sait, la première apparition en langue française d'une partie du Discours date de 1574. Publié sans nom d'auteur (le texte étant soi-disant composé par «Eusebe Philadelphe Cosmopolite»), mais attribué à Nicolas Barnaud, Le Réveille-Matin des François et de leurs voisins représente la première réception ouvertement politique - c'est-à-dire religieuse dans le contexte du conflit qui divise la France depuis 1563 - du Discours par les réformés. Quelques passages choisis, en fait remaniés et tronqués, furent pour l'occasion extraits du texte de La Boétie et présentés dans le contexte des événements contemporains dans le second dialogue. Lors de cette première récupération huguenote, le Discours de La Boétie fut amputé de presque $80 \%$. Il fut transformé et incorporé dans un pamphlet qui appelait à la sédition au lendemain du massacre de la Saint-Barthélemy; mais le court extrait du Discours restait néanmoins anonyme et ne semble pas avoir été immédiatement attribué à La Boétie. Le second dialogue du Réveille-Matin reproduit deux passages du Discours de la servitude volontaire (p. 178 et pp. 180-191) intégrés aux déclarations du «Politique» dans sa conversation avec l'«Historiographe François», alors qu'ils se dirigent tous deux vers une «hostellerie à Fribourg». Nicolas Barnaud traduisit pratiquement en même temps ce livre en latin sous le titre Dialogi ab Eusebio Philadelpho... (Edimburgi, ex typografia Iacobi Iamœi, 1574), ouvrage qui, apparemment, fut publié quelques semaines avant la version française. Comme on le voit, la première réception du Discours est donc indissociable de la cause protestante. 
Trois années plus tard, le Discours fit l'objet d'une seconde récupération au service de la cause protestante. En 1577, c'est en effet Simon Goulart, dans les Memoires de l'Estat de France sous Charles IX - plus connu sous le nom de Mémoires de Charles $I X^{6}-$, qui donna une véritable visibilité au Discours de La Boétie. En moins d'une année, trois éditions se succédèrent rapidement et le Discours acquit pour la première fois une circulation importante. C'est surtout dans sa troisième impression datée de 1578 que le Discours connut une large diffusion, qui influença pendant deux siècles la façon dont ce texte sera reçu et interprété. En effet, les trois éditions des Mémoires de Charles IX, toutes sous le nom d'emprunt de Heinrich Wolf, reproduisirent pour la première fois le Discours dans sa totalité. C'est dans le troisième volume des Mémoires qu'apparaissait le Discours au milieu d'autres documents pouvant servir à la rédaction d'une histoire du règne de Charles IX dans une perspective huguenote. Dans le contexte des autres documents rassemblés et des analyses de Goulart, le Discours - sans qu'il soit tronqué ou déformé - passe ouvertement pour un pamphlet protestant.

Quelques années plus tôt, alors qu'il venait de vendre sa charge au parlement de Bordeaux, Montaigne décida de tenir les textes politiques de La Boétie à l'écart de l'édition des œuvres de l'ami disparu qu'il venait de faire imprimer chez Federic Morel à la fin de l'année 1570, jugeant leur «façon trop délicate et mignarde pour les abandonner au grossier et pesant air d'une si mal plaisante saison» ${ }^{7}$. Il réservait l'exclusivité du Discours à un ouvrage du sien où le texte de La Boétie occuperait une place de choix parmi ses propres réflexions d'ordre diplomatique, moral et militaire, car il faut rappeler que les premiers Essais de 1580 portaient principalement sur des questions de politique et d'ambassade ${ }^{8}$. Pour Montaigne, le Discours de La Boétie s'insérait tout à fait dans le projet éditorial envisagé pour ses Essais, car il remplissait un critère essentiel, à savoir l'absence de références historiques explicites.

Alors que l'impression de la première édition de ses Essais à Bordeaux était sur le point d'être achevée, une nouvelle édition augmentée des Mémoires de Charles IX (édition dite à gros caractères) - en fait une contrefaçon de l'édition de 1578, dite à petits caractères -, vit le jour. Les répercussions politiques de cette publication furent immédiates et le projet de Montaigne se trouva irrémédiablement bouleversé. C'est en effet véritablement à partir de ce moment - donc en 1578 - que le Discours acquit une visibilité qui ne permettait plus d'incorporer ce texte aux Essais. Imprimée à fort tirage, cette édition pirate en trois volumes fut composée à partir de la troisième édition des Mémoires imprimée la même année. Le Discours de la Servitude volontaire représente 46 pages (f. 116v-139v) dans cette édition que l'on trouve encore aujourd'hui relativement facilement chez les libraires anciens. La

(6) Volume I: [*1r]: titre (verso blanc); *2r-*8r: «Au lecteur S.» (préface de Simon Goulart) $(* 8 \mathrm{v}$ blanc); f. 1r-655r: «Memoires de l'Estat de France sous le Roy Charles IX, depuis le troisiesme edict de pacification fait au mois d'Aoust 1570, jusques au commencement du regne de Henry III»; 4N7v-4O3v: «Indice des principales matieres contenues en ce premier volume» (index). Volume II: [II 1r]: titre (verso blanc); II2r-II4r: «Au lecteur S.» (préface de Simon Goulart) (II4v blanc); IT $5 \mathrm{r}-$ IT7v: «Indice des principales matieres contenues en ce deuxiesme volume des memoires de l'estat de France» (index); f. 1r-630r: «Memoires de l'Estat de France sous le Roy Charles IX. Volume second». Volume III: [A1r: titre (au verso l'éditeur précise qu'un index est ajouté à la fin de l'ouvrage); f. 2r-494r: «Memoires de l'Estat de France sous le Roy Charles IX. Volume troisiesme»; 3Q6v-3Q8v: «Indice des principales choses contenues en ce troisiesme volume» (index).

(7) Mesnagerie de Xénophon, dans Euvres complètes d'Estienne de La Boétie, éd. P. Bonnefon, Bordeaux/Paris, G. Gounouilhou/J. Rouam, 1892, «Advertissement au lecteur, par M. de Montaigne». Cet avertissement est daté du 10 août 1570.

(8) Voir Ph. Desan, Montaigne. Une biographie politique, Paris, Odile Jacob, 2014, plus particulièrement le chapitre VI: «Les Essais de 1580: discours moraux, politiques et militaires». 
table des matières présente le texte de La Boétie comme «Discours, de la servitude volontaire». Notons que le terme «Discours» ne semble pas faire partie du titre puisqu'il est suivi d'une virgule, ce qui le détache du titre qui suit'. On remarquera aussi que le texte du Discours n'est pas séparé du texte qui le précède puisqu'il ne commence pas sur une nouvelle page. De même, la fin du Discours ne se distingue pas facilement du commentaire de Simon Goulart qui lui fait suite et n'est indiqué que par une manchette en italiques, laquelle présente, sous forme de sommaire, la narration des événements historiques de la fin du règne de Charles IX. Il faut enfin préciser que c'est le texte de cette édition clandestine - parce qu'elle fut de loin la plus répandue - qui sera retenu pour toutes les réimpressions du Discours de La Boétie dans les œuvres de Montaigne publiées par Pierre Coste au XVIII siècle (1724, $1727,1739$ et 1745$)^{10}$.

La réaction envers cette édition fut immédiate en terre catholique. Par arrêt du parlement de Bordeaux, les Memoires de l'estat de France furent brûlés sur la place de l'Ombrière à Bordeaux le 7 mai 1579, deux jours avant l'obtention du privilège accordé à Simon Millanges pour les Essais de Montaigne et autres «nouvelletez». Il était soudain devenu impossible pour Montaigne de publier le Discours de La Boétie dans ses Essais en 1580 et de se voir ainsi associé à ce qui était désormais un pamphlet protestant. Au dernier moment, Montaigne se résolut à supprimer le Discours de son livre et le remplaça par vingt-neuf sonnets de La Boétie.

Montaigne tentera malgré tout d'expliquer que cet exercice de jeunesse ne correspondait nullement à ce que les protestants voyaient dans le Discours. Dans les années 1550, puis au début des années 1560, le Discours avait pu passer pour une réflexion universelle sur la liberté, une méditation affranchie de tout dogme et de toute contrainte politique. Montaigne privilégie cette explication et fait l'apologie d'un texte qui aurait échappé à l'intention première de son auteur. Selon lui, le Discours représente un texte de portée générale qui s'offre comme une réflexion sur la condition humaine. La Boétie s'était d'ailleurs livré à un exercice scolaire représentatif du milieu robin. En effet, de nombreux ouvrages rédigés par les plus grands jurisconsultes de cette époque, notamment les premières réflexions de Jean Bodin sur le droit universel, offrent eux aussi matière à «réflexion universelle sur la condition humaine ${ }^{11}$. Il ne faut donc pas confondre la culture robine du milieu du siècle avec les pratiques pamphlétaires du temps des guerres de religion. Pour cette raison, il est nécessaire d'établir une distinction entre la réception manuscrite du Discours durant les années 1550-1560 et sa "récupération" par les protestants dans les années

(9) Montaigne va dans le même sens quand il écrit que le texte de La Boétie «est un discours auquel il donna nom De La Servitude volontaire» (Essais 1580, I, 28, p. 252). Le titre est mis en italiques et ne laisse donc subsister aucun doute sur l'intitulé exact du discours de La Boétie. De plus, Montaigne semble accepter une lecture «protestante» du Discours en remarquant que «ceux qui l'ont ignoré, l'ont bien proprement depuis rebaptisé le contre un» (ibid.), reconnaissant ainsi un contenu essentiellement politique au Discours. Pourtant, en 1570, à l'occasion de la publication des œuvres de La Boétie chez Federic Morel, et donc avant la récupération de La Boétie par Nicolas Barnaud et Simon Goulart, il mentionne «un Discours de la servitude volontaire, E quelques memoires de noz troubles sur l'Edict de Janvier, 1562». L'avertissement au lecteur étant entièrement imprimé en italiques, il est donc difficile d'affirmer que «Discours» (avec une majuscule) fait bien partie du titre. Il est en effet assez courant de mettre «Discours» avec une majuscule dans les textes de cette époque.

(10) Protestant d'origine italienne, réfugié à Londres pour éviter les persécutions de l'Édit de Nantes, Pierre Coste sera le seul éditeur des œuvres de Montaigne durant la première moitié du XVIII ${ }^{\mathrm{e}}$ siècle. Une fois de plus, ce sera donc un protestant qui permettra au Discours de retrouver une nouvelle visibilité au siècle des Lumières.

(11) J. Bodin, Juris universi distributio, dans Euvres philosophiques de Jean Bodin, éd. P. Mesnard, Paris, Presses Universitaires de France, 1951, pp. 71-80 [Tableau du droit universel, pp. 83-97]. 
1570. Du manuscrit à l'imprimé, l'interprétation même du Discours se trouva sensiblement modifiée. Le Discours était maintenant devenu un factum au service de la cause huguenote, quoi qu'en dise Montaigne.

Selon Montaigne, La Boétie aurait écrit le Discours «par maniere d'essay», en sa première jeunesse. Sur l'Exemplaire de Bordeaux, l'auteur des Essais rajeunit La Boétie de deux ans: «dix-huit» est biffé et remplacé par «sèze», ce qui ferait remonter la première rédaction du Discours aux alentours de 1546, donc avant la révolte de la Gabelle. Ce rajeunissement de La Boétie n'est certainement pas innocent, car Montaigne prend soin de déshistoriciser le Discours et le présente comme un texte général «a l'honneur de la liberté contre les tyrans» ${ }^{12}$. L'usage du terme «essai» pour définir le Discours permet à Montaigne d'établir un lien direct avec son propre ouvrage, mais il précise aussi le but politique de la réflexion de son ami, c'est-à-dire une pensée inachevée et conséquemment ouverte à l'interprétation. Dans ce passage, Montaigne fournit plusieurs clés de lecture du Discours. Tout d'abord, il souligne l'aspect spéculatif et abstrait de ce texte qui, selon lui, ne peut être confondu avec d'autres écrits plus ponctuels qui renverraient aux événements politiques depuis 1572, bien qu'ils soient à première vue de même "farine". On voit ici que Montaigne joue dans l'ambiguité, choisissant d'émettre une opinion sur l'origine et le contenu du Discours avant de dire le contraire quand il aborde le texte de La Boétie dans son existence imprimée, la seule connue par une grande majorité du public après 1578 .

Il ne faut pas s'y tromper, ces paroles qui visent à réduire l'importance politique et idéologique du Discours sont elles-mêmes conditionnées par l'incident de l'autodafé public des Mémoires de Charles IX devant le parlement de Bordeaux peu de temps avant la publication des Essais. Montaigne s'empresse d'ajouter que ce «subject» ne représente pas un simple exercice - contrairement à ce qu'il venait d'affirmer un peu plus tôt - puisque le jeune La Boétie croyait dans les thèses avancées. Il précise que son ami avait pour principe de toujours obéir et de se soumettre «tresreligieusement» aux lois de son pays. Il prend ensuite le contrepied avec les thèses avancées par La Boétie et entre en contradiction avec sa propre déclaration en associant le Discours à un Contr'Un. Ce second titre proposé pour le Discours ne laisse subsister aucun doute sur la façon dont il doit être interprété à son époque. De plus, le nom de Contr'Un ne se trouve que chez Montaigne. En proposant un nouveau titre, Montaigne résume en quelque sorte le contenu essentiellement politique du Discours et pose par la même occasion la question de l'allégeance dans son rapport à la servitude. Peut-être malgré lui, Montaigne souligne la dimension politique - et contemporaine - du Discours. Il semble contredire ses déclarations antérieures en allant jusqu'à proposer un nouveau titre qui correspond mieux à l'actualité politique de son temps.

Peut-on à la fois faire l'apologie de la liberté et se plier en même temps aux lois «injustes» du pays - Montaigne préfère le mot «patrie» - dans lequel on est né? Estil possible de développer un discours sur la liberté et l'affranchissement de l'homme tout en acceptant ce que Descartes appellera, dans le Discours de la méthode, une «morale par provision», c'est-à-dire une morale qui se présente comme un universel dans un espace culturel déterminé et ne peut être remise en cause sur le plan politique, culturel et social? Le principe régulateur avancé par Montaigne est en fait celui d'un équilibre à trouver entre coutume et liberté. Ce thème si cher à Montaigne est déjà présent dans le Discours de La Boétie. Selon l'auteur des Essais, et en accord avec La Boétie, les pratiques religieuses ne relèvent pas de l'entendement 
humain: «Nostre foy ce n'est pas nostre acquest, c'est un pur présent de la libéralité d'autruy. Ce n'est pas par discours ou par nostre entendement que nous avons receu nostre religion, c'est par authorité et par commandement estrangier» ${ }^{13}$. Ce passage se présente comme un dialogue avec les protestants, voire une réponse aux Monarchomaques.

Montaigne aurait donc dû être le premier éditeur du Discours de la servitude volontaire, mais il se fit prendre de court par Simon Goulart. L'auteur des Essais possédait pourtant le Discours sous forme manuscrite depuis plus de quinze ans. C'est donc avec beaucoup de retard qu'il jugea nécessaire de faire imprimer le texte de l'ami disparu. On sait à ce sujet que Montaigne connut La Boétie tardivement - probablement durant l'année 1559 -, c'est-à-dire bien après la rédaction du Discours. Au début des années 1560, La Boétie passait pour un fervent défenseur du pouvoir parlementaire et s'opposa au pouvoir militaire et politique des gouverneurs. En décembre 1561, son nom apparaît par exemple sur une liste de douze conseillers chargés de diverses mesures de police, notamment de désarmer les tenants de la nouvelle religion. Le parlement de Bordeaux était encouragé par les succès militaires de Monluc, gouverneur de Guyenne en remplacement du duc de Montpensier, qui passait à juste titre pour un redoutable militaire toujours prêt à en découdre avec les séditieux ${ }^{14}$. Le Mémoire sur l'édit de Janvier fut rédigé à cette époque, c'est-à-dire au temps où La Boétie croyait encore à la mise au pas des «religionnaires». Montaigne séjournait dans la capitale en cette fin d'année 1561. En juin 1562, de sa propre initiative, il fit profession de foi catholique devant le parlement de Paris. Il partageait pleinement les vues des parlementaires bordelais qui soutenaient la répression catholique contre les partisans de la nouvelle religion. Comme La Boétie, il fut par exemple opposé à toute tolérance religieuse, car il concevait mal comment deux religions pourraient coexister dans un même pays. Cette position reflète le point de vue de la grande majorité des parlementaires à Bordeaux au début des années 1560. En fait, Montaigne et La Boétie défendaient une position idéologique qui s'apparentait à une forme de corporatisme robin. En 1561 - comme Montaigne dans les années 1570 -, La Boétie recommandait quant à lui la modération, mais pas la concession. Évidemment, à la fin des années 1570, les guerres civiles avaient enseigné à Montaigne que l'édit de Janvier - et toute politique de modération en général - n'avait strictement rien changé aux troubles qui sévissaient en France. L'actualité des années 1570 avait donné une nouvelle vie au Discours qui évoquait de façon abstraite et théorique des questions devenues ponctuelles et pratiques.

Influencé par la réflexion des Monarchomaques sur l'absolutisme royal, Montaigne s'interrogera à son tour sur le lien qui unit le roi et ses sujets. La Boétie avait également tenté d'imaginer le rôle du peuple affranchi dans cette redistribution des pouvoirs: le peuple se laisse asservir par le simple fait qu'il ne choisit pas d'être libre. Vouloir l'être représenterait déjà une forme de liberté. Cette conception de la liberté ressemble plus à une liberté de conscience (terme redéfini par les pratiques religieuses du temps) qu'à une véritable liberté d'action, et l'on comprend comment le Discours a pu logiquement être récupéré par les huguenots qui avaient d'abord besoin d'établir cette forme abstraite de liberté avant de passer à l'étape suivante de l'action politique et de la résistance armée. Comme on se plaît à le faire remarquer à partir des années 1570, à quoi servirait d'ailleurs la liberté de pensée sans son expres-

(13) Montaigne, Essais 1580, II, 12, p. 257.

(14) C.-B.-F. Boscheron Des Portes, Histoire du parlement de Bordeaux, Bordeaux, Charles Lefebvre, 1878 , p. 166. 
sion et son application concrète sur le plan politique? Dans le Discours, La Boétie ne présente pas l'argument des Monarchomaques pour qui la liberté de conscience ne peut exister sans une liberté de culte. C'est pourtant ce qui permit à son traité de passer à la postérité. Le Discours offrait désormais le fondement théorique pour un appel à l'action militaire. On pourrait dire que La Boétie fut en quelque sorte dépassé par son Discours.

$$
* * *
$$

On voit poindre chez La Boétie la pierre angulaire du libéralisme moderne: la liberté individuelle détachée de toute action politique ou sociale. La Boétie ne recommande jamais l'action (le soulèvement par exemple) contre la servitude et il rejoint sur ce point essentiel la position de Luther dans son célèbre texte sur la liberté chrétienne ${ }^{15}$. Ainsi, dans son Commentaire sur l'Épitre aux Galates (1531-1535), Luther donne une définition limpide de la liberté chrétienne:

Il s'agit de la liberté par laquelle Christ nous a affranchis, non de telle ou telle servitude humaine, ou du pouvoir des tyrans, mais de la colère éternelle de Dieu. Où [est cette liberté]? Dans la conscience. C'est là que demeure notre liberté: il ne faut pas chercher ailleurs. Car ce n'est pas d'une liberté politique dont Christ nous a fait don en nous affranchissant, et ce n'est pas une liberté charnelle. C'est théologiquement et spirituellement qu'il nous a affranchis, c'est afin que notre conscience soit libre et heureuse, n'ayant nulle crainte de la colère à venir [...]. C'est une liberté ineffable que d'être affranchis éternellement de la colère de Dieu: une liberté plus grande que le ciel et la terre et que toutes les créatures ${ }^{16}$.

Cette liberté ne délivre donc pas de l'assujettissement ou de la servitude et ne conduit jamais à une amélioration sociale et matérielle de l'existence humaine. La théorisation d'une liberté au seul niveau de la conscience était déjà défendue par Luther en 1525 dans sa réponse aux paysans qui demandaient que cette "liberté dans les têtes" trouve une application concrète dans leur vie quotidienne. En effet, il est à notre avis possible d'établir un parallèle entre les idées défendues dans le Discours de La Boétie et la célèbre Exhortation à la paix à propos des douze articles de la paysannerie souabe (1525). Dans ce texte, Luther dissocie liberté et possibilité d'agir.

Dans la première partie adressée aux princes et aux seigneurs, Luther rappelle qu'il est de leur côté et les a toujours défendus contre la «populace insensée»: «Vous et tout le monde devez me rendre ce témoignage que j’ai enseigné dans le plus grand calme, que j'ai lutté avec force contre la révolte, que j'ai tenu à exhorter avec le plus grand zèle les sujets à l'obéissance et au respect, même à l'égard de votre autorité tyrannique et furieuse, de telle sorte que cette révolte ne saurait m'être imputée» ${ }^{17}$. Il admoneste ensuite les paysans à qui il reproche de vouloir se soulever. Sur un ton conciliant, Luther admet que les princes, en faisant peser des charges insupportables sur le peuple, «ont bien mérité que Dieu les fasse tomber de leur trône». On retrouve la même image chez La Boétie qui pense également que le tyran tombera

(15) Martin LuTHER traite plus particulièrement de la liberté chrétienne dans De la liberté du chrétien (1520) et dans son Commentaire sur l'Épitre aux Galates (1531-1535). Pour une présentation du débat sur la liberté dans la première moitié du XVI siècle, voir M. LiEnHARD, Le débat sur la liberté au temps de la Réforme, «Positions Luthériennes», vol., 37, 1989, pp. 323-339.

(16) M. Luther, Commentaire sur l'Épitre aux Galates, dans Euvres, 18 vol., Genève, Éditions Labor et Fides, t. XVI, p. 178.

(17) M. LuTHER, Exhortation à la paix à propos des douze articles de la paysannerie souabe, dans Euvres, ibid., t. IV, p. 151. 
tout seul de son piédestal si notre conscience est libre. Surtout ne pas le pousser, ne pas l'ébranler, il suffit simplement de ne pas le soutenir! La liberté se résume à une libre pensée et refuse toute possibilité d'action. De même, trente ans plus tôt, Luther rappelait aux paysans que seule une «bonne conscience» suffisait pour que Dieu les assiste: «Et même si, pendant un temps, vous aviez le dessous et enduriez la mort pour cela, vous n'en finirez pas moins par gagner et votre âme subsisterait éternellement avec tous les saints» ${ }^{18}$. Luther évoque le Christ qui, selon lui, aurait toujours défendu le principe de ne «pas résister au mal et à l'injustice, mais toujours céder, souffrir et laisser prendre» ${ }^{19}$. «Souffrance, souffrance, croix, croix, voilà le droit des chrétiens, cela et pas autre chose!» s'exclame Luther. Rien ne sert de se soulever pour des biens temporels. Un bon chrétien doit se résigner et attendre que les idoles s'écroulent par elles-mêmes. Il recommande aux paysans souabes de laisser «votre poing et votre épée, votre mutinerie et vos menaces» ${ }^{20}$ et leur reproche de vouloir faire de tous les hommes des égaux, ce qui est pour lui impossible, «[c]ar un royaume temporel ne saurait exister s'il n'y a pas d'inégalité entre les personnes, en ce sens que certains sont libres et d'autre enchaînés, certains, seigneurs, et d'autres, sujets» ${ }^{21}$. Pour Luther, la liberté chrétienne ne réside pas dans le temporel; chaque homme est libre au niveau de sa conscience et non de son existence.

Être libre c'est désirer l'être, mais surtout pas prendre les armes pour s'affranchir du joug de l'oppression des nobles. Être libre est plus un état d'esprit qu'une réalité matérielle pour Luther. Dans le Discours, La Boétie est également catégorique sur ce point et semble se rallier à la position de Luther: il s'agit pour le peuple «non pas de [se] delivrer, mais seulement de le vouloir faire» ${ }^{22}$. La liberté se résume à une question de volonté. C'est là une distinction assez extraordinaire, car elle dissocie la théorie de la pratique. La Boétie explique ce qu'il entend par liberté et précise les limites de toute action contre le maître ou le tyran: «Je ne veux pas que vous les poussiés ou l'esbransliés, mais seulement ne le soustenés plus, et vous le verrés, comme un grand colosse à qui on a desrobé la base, de son pois mesme fondre en bas et se rompre $\gg^{23}$. L'apologie de l'inaction par le retrait engendre pourtant un problème moral. Doit-on se taire ou se révolter - en paroles, mais aussi par les armes - contre la tyrannie?

Montaigne prendra cette recommandation de La Boétie comme point de départ pour réfléchir à son tour au lien qui unit le maître au serf ou, dans son cas, le lien qui unit le patron à son client, le maître au serviteur. Il aurait pu citer le début du Discours où La Boétie exclut tout rapport social durable entre deux êtres: «[C]'est un extreme malheur d'estre subject à un maistre, duquel on ne se peut jamais asseurer qu'il soit bon, puisqu'il est tousjours en sa puissance d'estre mauvais quand il voudra ${ }^{24}$. L'allégeance doit-elle être indépendante de la bonté du mâtre ou du monarque quand la magnanimité du prince est essentiellement contractuelle (dans la mesure où chaque parti profite de l'allégeance comme de la servitude)? À vrai dire, une telle remarque sur les conséquences de la servitude n'est guère utile pour comprendre ce qui pousse les hommes à abandonner une partie de leur liberté pour

(18) Ibid., p. 153.

(19) Ibid., p. 158.

(20) Ibid., p. 162.

(21) Ibid., p. 167.

(22) ÉTIENNE DE La BoÉTIE, De la servitude volontaire ou Le contr'un, éd. N. Gontabert, Paris, Gallimard, 1993 , p. 88.

(23) Ibid.

(24) Ibid., p. 79. 
accéder à une position matérielle stable au sein d'un réseau social et politique où la corruption et l'exploitation ne peuvent jamais être exclues. L'allégeance sous-entend une attente et un gain anticipé, mais rien n'est jamais certain. Sur ce point, la première partie du Discours est empreinte d'un idéalisme déconcertant, un idéalisme de la liberté qui à notre avis représente une conception fondamentalement libérale et moderne de la liberté.

Le Discours de La Boétie eut sur ce point une influence considérable sur Montaigne. C'est précisément le rapport problématique entre servitude (terme trop abstrait et idéalisé) et allégeance (terme qui induit une structure d'obligations réciproques et de gains mutuels) qui conduisit Montaigne à distinguer deux formes de liberté: l'une théorique (souvent exprimée par l'essayiste en tant qu'individu privé - Michel de Montaigne -, maître de son jugement), et l'autre, plus pratique, qui correspond à l'homme public (le maire ou le négociateur) dans un cadre plus large, défini par des rapports de pouvoir, des obligations et des allégeances qui permettent au sujet d'exister socialement. Tous les hommes disposent de leur liberté individuelle dans la sphère privée - cette remarque procède d'une approche qualitative -; mais sur le plan public Montaigne défend le statu quo et l'inaction. C'est pour cette raison qu'il a souvent été considéré - justement - comme un conservateur.

La solution préconisée par La Boétie pour émanciper l'homme de sa «servitude volontaire» n'est pas d'appeler à l'organisation ou au rassemblement d'individus au sein d'un groupe politique afin de passer à l'action; elle ne débouche pas sur la formation d'une collectivité rebelle. Au contraire, il privilégie le développement d'une forme d'amitié entre le maître et le subordonné. Cette amitié fusionnelle sera reprise par Montaigne; elle procède par absorption mutuelle de l'un dans l'autre, non par une union ou un regroupement de l'un avec l'autre. La distinction est subtile, car cette liberté, écrit La Boétie, «ne vouloit pas tant nous faire tous unis que tous uns, il ne faut pas faire doute que nous ne soions tous naturellement libres, puisque nous sommes tous compaignons, et ne peut tomber en l'entendement de personne que nature ait mis aucun en servitude, nous aiant tous mis en compaignie $\gg^{25}$.

À la place grandissante donnée à la raison s'oppose l'empire de la coutume. Ce fondement de la pensée montaignienne est déjà clairement exprimé par La Boétie qui n'hésite pas à avancer que la forme définit le contenu et que l'homme «tient le pli que la nourriture lui donne» ${ }^{26}$. Dans un passage profondément montaignien, La Boétie va même jusqu'à affirmer que la coutume est seule responsable de l'organisation politique (et donc de la servitude volontaire qu'il vient de décrire) dans les sociétés humaines: «[À] l'homme toutes choses lui sont comme naturelles, à quoy il se nourrit et accoustume [...] ainsi la premiere raison de la servitude volontaire, c'est la coustume ${ }^{27}$. Montaigne reprendra à son compte cet argument en affirmant que l'on ne peut effectivement pas se débarrasser de la coutume pour concevoir de nouveaux systèmes politiques ou religieux, et qu'il est impossible de vivre hors de la culture dont nous avons hérité.

Doit-on en déduire que la servitude, comme l'idéologie, est inévitable? Parce que l'homme est un être social, la solution proposée par La Boétie (et Montaigne après lui) consiste à être conscient - et seulement conscient, sans vouloir pour autant changer la société ou le monde - de sa condition humaine. Il ne s'agit pas encore de proposer un contrat social, dans l'acception rousseauiste du terme, mais simplement 
de reconnaître la condition matérielle et historique de l'homme par l'acceptation obligée d'une morale et des institutions politiques et religieuses en place, faute de mieux. On naît catholique et Français comme le rappelle Montaigne: c'est là une forme de servitude volontaire, obligatoire et inévitable, dans la mesure où la religion et la politique résultent de la coutume. La coutume est nécessairement volontaire puisqu'elle s'impose à tous de la même façon. Nous ne pouvons que l'embrasser. Autant dissocier liberté de pensée et soumission aux règles et aux lois qui font de nous des êtres sociaux. Il est alors logique que la liberté soit une forme de servitude volontaire pour La Boétie et Montaigne. Elle ne résulte pas d'une métaphysique, mais d'une sociologie, car son fondement relève bien du politique et dépasse les choix personnels. C'est de cette façon que l'allégeance aux règles établies par d'autres ne s'oppose pas à une liberté de principe. Etre libre, c'est conserver la possibilité de son affranchissement, tout en se pliant aux lois qui nous contraignent à la servitude. Cette possible liberté tient lieu de liberté pour La Boétie, comme pour Montaigne, et préfigure sur bien des points les thèses de John Locke sur la liberté individuelle.

Pour La Boétie, la conséquence de la servitude volontaire n'est pas tant l'abandon du libre arbitre de chacun, que la manifestation possible de la tyrannie. Après tout, la servitude volontaire n'empêche pas le fonctionnement de la société; la tyrannie représente par contre le degré inacceptable d'un mal nécessaire. Elle est pour lui la limite à ne pas dépasser dans la pratique du pouvoir. Une fois ce seuil franchi, le contrat qui lie le prince au peuple est nécessairement rompu. C'est bien de cette façon que le Discours sera interprété par les protestants dans les années 1570. La tyrannie transforme définitivement le pouvoir d'un seul sur tous dans la mesure où l'individu ne peut plus tirer profit de son assujettissement; elle remet en cause l'idée même de servitude volontaire (ou, plus précisément, d'allégeance). Ce constat fait surgir la question du soulèvement armé dans les textes protestants des années 1570, notamment dans les Vindiciae contra tyrannos de Duplessis-Mornay. En défendant cette position, La Boétie ne pouvait imaginer les événements sanglants des premières guerres civiles, mais, vingt ans plus tard, cette même question de la tyrannie était d'une actualité brûlante.

Ceux qui avaient récupéré le Discours de La Boétie dans les années 1570 firent valoir une théorie de la représentation politique s'appuyant sur les magistrats - limités en nombre - dont la fonction aurait été de servir de contre-pouvoir au souverain. Ces élus de Dieu sont en quelque sorte les garants de la liberté humaine et leur rôle consiste à s'opposer à toute forme de soumission au tyran. Sur ce sujet, et comme par anticipation des théoriciens politiques des années 1570, La Boétie remarque ainsi qu'il se trouve «quelques uns, mieulx nés que les autres, qui sentent le pois du joug et ne se peuvent tenir de le secouer; qui ne s'apprivoisent jamais de la subjetion ${ }^{28}$. On comprend comment Simon Goulart, Duplessis-Mornay et autres penseurs proches des huguenots ont pu reconnaître dans ces «quelques uns» les magistrats «aians l'entendement net et l'esprit clairvoiant», les élus de Dieu qui ne se contentent pas, «comme le gros populas», de suivre ceux qui les mènent dans un état de servitude volontaire. Ces individus choisis et élus par Dieu ne possèdent pas seulement (et l'on retrouve ici une expression chère à Montaigne) «la teste [...] bien faite», mais «ancore polie par l'estude» ${ }^{29}$. Lu dans le contexte religieux de l'aprèsSaint-Barthélemy, le Discours avait soudainement acquis une dimension politique séditieuse qu'il ne possédait pas forcément lorsqu'il avait circulé parmi un petit 
cercle de robins initiés aux valeurs humanistes sur la liberté universelle. Comme Luther avant lui, La Boétie avait défendu une liberté plus théorique que pratique, mais les Monarchomaques et autres penseurs réformés virent dans cet «exercice de jeunesse» un texte leur permettant précisément de transcender la simple liberté de conscience pour appeler au soulèvement armé, non pas en faisant appel au peuple, mais en s'appuyant sur le pouvoir des magistrats mis en évidence dans le Discours ${ }^{30}$.

PHILIPPE DESAN

University of Chicago

(30) Une version abrégée de cette étude a été présentée lors de la «Journée d'étude sur le Discours de la servitude volontaire de La Boétie» organisée par Déborah Knop et Jean Vignes le 28 novembre 2014 à l'Université Paris 7 Denis-Diderot. 